\title{
Research for Problems of China College Entrance Examination of Sports Majors and Countermeasures
}

\author{
Du Weiwen \\ Sports \& Art College, Hunan Agricultural University, Changsha, Hunan, China \\ Corresponding author email: $582110392 @ q q . c o m$
}

\begin{abstract}
The purpose of the test o professional sports is to select qualified sports talents. It relates to the quality of training talents across the century. With the development of the times, in face of the challenges of the new era, the reform of physical education becomes urgent besides the implementation of humane concern for students. This paper tries to discuss the content and evaluation standard of professional sports tests and put forward ways of applying the equipment of new standardized automatically measuring instruments, the cancel of profession tests held by schools, the provincial test with student's self selection and so on, so as to establish dynamic items of professional sports tests with characteristics of time, regions and nationalities, thus meet the needs of new trend of the development of sports.
\end{abstract}

KEYWORD: China College Entrance Examination of Sports Majors; Problem; Countermeasure

\section{PROBLEMS}

Enrollment work examination professional sports in China has been practiced for many years, scientific, standardized, standardized measures for the implementation of a set of authoritative higher, however, with the development of the times, the progress of the society and the continuous deepening of China's sports socialization, face the challenges of the new century, the problems and lag behind the development the tide and trend of sports of our country sports specialty is the college entrance examination in the emerging [1-4], (Wang Bin and Li Xiaoping, 1996; Shao Minh et al, 1998; Zhou Guoru, 1999 and $\mathrm{Hu}$ Yuanming of Hubei province, 2009), such as all kinds of professional exam, each area and each area of each school are act of one's own free will, it is The Eight Immortals Crossing the Sea, each shows special prowess, this kind of serious gave birth to the sports oriented phenomenon and hidden as the behavior, the school sports teaching greatly deviate from "the basic direction to students' health oriented", seriously affected the physical and mental health of students and interested in sports.

As everyone knows, sports specialty is sports professional college entrance examination examination, performance will directly influence whether candidates can be admitted, sometimes 1 points to the examinee can cause lifelong regret, the fierce competition is one can imagine, you do things by irregular ways attitude often manifested in the examination process, candidates will know the exam from the loophole each aspect, adopt various illegal means to attempt to improve their test scores. If the examinee by changing the photo, making false admission ticket, identity card or let looks very like the double (multiple) twin brothers and sisters, "Gunners" generation test, doping eat, drink drink "doping" so as to improve the result of the examination, cheating is full of tricks, shocking. Combined with the characteristics of sports exam itself, not like the culture of college entrance examination, to achieve sealing marking, nuclear, and must be based on pre programmed "order list" for the examination, let some parents eager to the various relationships become an unwholesome tendencies, unable to use the examiners and candidates and parents barrier the exchange of students and their parents, especially, the team of teachers and teachers in business center, the academic links, to the examinee examination grades applied anthropogenic effects [5-6] (Luo Fang, 2006; Chen Qingwei 2012)

In addition, the sports professional examinations are outdoors, experiencing a continuous rain or high wind, causing the ground muddy, slippery; influence sometimes need to change the site or delay the test time and other objective factors, the candidates can not play to the best state, also, the examiner will often under the scorching sun or rain work, easy due to fatigue and make results appear error [5]. (Luo Fang, 2006) 


\section{COUNTERMEASURES}

Sports professional examination organization management scientific not only directly affect the selection of talents in higher school physical education professional, but also related to students' future, affecting thousands of households, public concern and attention of [5] (Luo Fang, 2006), so this review examination of professional sports, Ying Yijian Kang development to ensure that, in order to avoid manipulation and examination is not perfect in part, let examinee drilled weaknesses, I put forward the following suggestions:

\subsection{Model standard automatic measuring instrument modification}

For the high jump, long jump, shot put measurement, softball results, as long as the good grades by instrument end point, automatic laser remote measurement, connect the starting point and achievement, and computer software, on the spot to read aloud the achievements, let the examinee and the referee to see the authenticity, accuracy of the results.

For running, whether it is running or dash, at the beginning, as long as the referee or by remote control or running special table, it can be directly issued orders: "On your marks!, preparation, gunfire", and instrument at the starting point according to the measuring instrument and the end is the Become (i.e. one to two), the instrument can also according to the number of running to set up, arrive at the end of every athlete, as long as the referee or press or remote measuring instruments, measuring instruments reported immediately to the athletes reach the end result, even if separated by short time distance instrument can distinguish. This can avoid sometimes shots not started and the correctness of the starting time of each control in equal time and achievement.

Used for basketball, volleyball, football, rope skipping and so when testing the countdown timer, start a test, the referee or by remote control or issued a directive, countdown table immediately began to whistle, like basketball, volleyball, football is break, at rest at the beginning, the referee or press or the remote controller sends a command, a countdown meter immediately stayed there without moving, and rest before beginning, referee or by remote control or issued a directive, countdown table immediately followed, when the test was over, the countdown table immediately issued at the end of the whistle, and can read aloud each performance test. As for skipping test did not break, use a countdown meter without intermediate stops, directly from the beginning to the end. Of course, these tests are available with a countdown timer, but test time according to the test requirements set.
Automatic measuring instrument using standard, can reduce the influence of human factors, the results of the candidates by the instrument automatically generated automatically saved, and can not be changed, the spot and report the athlete scores, improve the examination of the transparency, openness. Let the examinee to concentrate on, get rest assured. In addition, the use of electronic measuring disk device, and greatly reduce the work intensity test house, to reduce the impact of human factors, so the physical examination more fair, and the modification of the automatic measuring instrument is also applied to the various games.

\subsection{Professional achievement to cancel school exam, the province couplet plus student favourite work}

Now our country University projects related to recruit professional sports and test method by the test to determine their own universities, provinces, regions and local schools examination time, examination contents, examination requirements are not the same..... Not only the existence of the fractional difference area, more examination project is different and other unfair examination. In addition to the increase in the number of candidates registered for the professional sports, professional sports exam time with elongated, generally for 1 months or more, and local climate in China during the annual examination of April 3 a constantly changing, the weather suddenly the rain, the temperature fluctuated. The weeks, sports professional examinations are outdoors, the examiner also often to the scorching sun or rain test, very easy to cause and effect of the accuracy of ratings of work fatigue. The sports specialized examinee all have professional examination before the college entrance examination test run around here and there culture, are more likely to be too busy to be scorched by the flames.

In order to reduce the pressure of college entrance examination, especially to relieve the pressure of college entrance examination sports specialty is small, encourage some of the best students in sports skills learning, to complete the implementation of quality education, talent cultivating comprehensive selection, maintenance admission examination of justice, promote the comprehensive development of students, provide multiple choices for students' growth, it has become the inevitable requirement the educational reform and social development. Taking into account the college entrance examination including small college entrance examination sports specialty wide range, high sensitivity, strong policy, the reform should actively and steadily, in an orderly way, to actively explore a new mode of operation, to better meet the needs of a serious including sports specialty, college entrance examination. 
Recommendations in the annual fine dry, cool autumn weather at the same place, the items and requirements of professional sports college entrance examination (such as college entrance sports examination score $=$ physical exam scores + non special test of basic skills score + special skills test scores, and each item score by 1 compulsory subjects and 1 examination subjects), conditional provinces and various indoor test items and test, the examinee sports specialized test individual achievement full video preserved, put in the examinee admitted to Annex 1. As long as the exam passed, according to the different professional sports, and selection of different candidates, is the emphasis on physical or non special skill or special skills examination, also participated in the exam, there are more than two times the project performance, the high score, score is valid for 3 years. Conditional school, can also be students training candidates favourite workindividual full photograph video performance in the professional examination of the appendix to Annex 2 , as a supplement to exam results of professional sports. Such candidates to cheat phenomenon is avoided, the professional school exam is cancelled, a reasonable professional examination method was [5] (Luo Fang, 2006).

\section{CONCLUSION}

Sports professional examination is designed to select qualified sports talents, related to the quality of training talents across the century, with the development of the times, facing the challenges of the new century, in addition to the implementation of human care of candidates [5] (such as put some preparatory activities before the test of music, let the students be prepared after moving outside measurement) trend, reform lags behind the development of physical education has be imperative, content and evaluation standard of physical test items should be based on continuous complement, perfect, dynamic process to test program set shows regional, age and ethnic characteristics [2] (Shao Minh et al, 1998), new trend constantly with development of sports science

\section{REFERENCES}

[1] Wang Bin, Li Xiaoping (1996). A physical examination for Anhui sports science and technology. 3: 60-62.

[2] Shao Minhe, Wang Jian, Yang Xiaoai et al (1998). College physical education students majoring in physical education test project settings on J. Tianjin University of Sport. 1: 65-69.

[3] Zhou Guoru(1999). The physical education major student research and research policy issues relating to. J. Shenyang Sport University. 4: 35-37.

[4] Hu Yuanming of Hubei province (2009). Analysis and Countermeasur es of the China Three Gorges University physical education college entrance examination of journal. 31:297-298.

[5] Luo Fang (2006). Research on some problems of scientific management in P.E. recruitment examination Journal of Harbin Institute of Physical Education. 5: 37-39.

[6] Chen Qingwei(2012). PE test and pre analysis and countermeasure research of teaching and management, 28: 83-85. 